\title{
A framework for end-of-life photovoltaics distribution routing optimization
}

\author{
Qi Guo ${ }^{1 *}$ and Hongjun Guo ${ }^{2}$
}

\begin{abstract}
Transportation cost accounts for a significant portion among the total cost of photovoltaics (PV) recycling. The transportation cost can be significantly reduced with a well-planned vehicle routing. A generic mathematical framework was developed to generate the optimal distribution scheme for transporting retired PV. A static and a dynamic recycling algorithms were created as the optimization tool in the framework. As for the output, the framework generates the optimal distribution scheme, the transportation cost of the optimal scheme, as well as the amount of End-of-life PV that each PV recycling center receives. A case study was included to test the effectiveness of the proposed framework. The optimal costs generated by two algorithms were compared with the baseline cost. As a result, the cost can be reduced by 53\% when compared with the baseline. In addition, the static algorithm can obtain a result with decent accuracy and low computational cost.
\end{abstract}

Keywords: Photovoltaic recycling, Mathematical modeling, Route optimization

\section{Introduction}

In 2015, 50.6 GW new solar photovoltaics (PV) were installed worldwide. A $613 \mathrm{GW}$ grid-connected solar power was forecasted by the end of 2019 all over the world [1]. The amount of PV installed in the United States in 2016 achieved 14.7 GW, doubling the amount of the year 2015 . With the 39\% renewable electric occupancy, solar energy has become the most used renewable electric energy in the United States in 2016. As the largest installer, in 2016, PV installation in California accounted for $35 \%$ of the United States. The cumulative PV installation in CA has researched $17 \mathrm{GW}$ [2].

Due to containing toxic and value materials, all on site PVs need to be recycled after they retire in about $25 \mathrm{yr}$. [3]. However, recycling is less economical than disposing at present. Cost effectiveness has been emphasized as the greatest challenge while performing PV recycling [4].

Reducing the transportation cost is an effective way to reduce the cost of PV recycling. The transportation cost has been concluded as the leading cost while simulating the PV recycling process in Germany [5]. While planning EoL PV material recovery in New York, the transportation

\footnotetext{
* Correspondence: guoqi2007@qq.com

'Department of Engineering Technologies, Bowling Green State University, Bowling Green, Ohio 43402, USA

Full list of author information is available at the end of the article
}

cost was also concluded as an indispensable parameter in the recycling framework [6]. The proportion of the transportation cost among the total cost was also emphasized while performing a financial analysis of end-of-life (EoL) PV recycling in Italy [7]. Therefore, in order to make the recycling affordable and profitable, the transportation cost needs to be reduced [8].

Implementing mathematical optimization is an effective way to reduce the transportation cost. Classical mathematical optimization models of the vehicle routing for the facility location decision has been summarized [9]. A waste collection optimization was performed by minimizing the route distance. By optimizing, the average waste collection efficiency was improved by $37 \%$, and the fuel cost was reduced by $48 \%$ [10]. An optimization on solid waste collection and transport was performed by using the ArcGIS Network Analyst tool. As a result, a $48 \%$ gas saving was achieved on the optimal transport scenario [11].

As for the optimization algorithm, Mixed Integer Linear Programming (MILP) and Greedy Algorithm (GA) have been used broadly on mathematical models. In order to solve a EoL vehicle recovery problem in Turkey economically, a MILP optimization model was developed to minimize the costs on opening facilities, recovery processes, and transportation [12]. MILP was also applied on vehicle routing problems to maximize 
the on-time possibility with the shortest path. Some advanced GAs were developed on top of the original GA to solve specific problems. A modified GA was employed to solve the stochastic control problem on the networked storage operation area [13].

Though many mathematical optimization researches have been done, there is not an algorithm that can be directly used to minimize the transportation cost in PV recycling. The paper focuses on developing a generic mathematical optimization framework to facilitate EoL $\mathrm{PV}$ recycling to reduce the transportation cost. Two recycling algorithms were proposed in the framework. A case study was included to show the efficacy of the framework.

\section{Materials and methods}

A mathematical modeling framework was developed to generate the optimal distribution scheme by minimizing the transportation cost. In the framework, three parameters are included: 1) Photovoltaics Installation Site (PVIS); 2) Photovoltaics Recycling Center (PVRC); and 3) Transportation Company (TC). This is typically a combinatorial optimization problem: each PVIS can be taken by any of TC, and sent to any PVRC. As a result, the optimal distribution scheme is presented by showing which PVIS is transported by which TC, and goes to which PVRC. The maximum annual capacities of each PVRC and TC are constraints to affect the distribution decision. Besides, the total transportation cost of the optimal distribution scheme can be calculated.

Three types of input data are required in the framework. These data are the transportation cost charged by TC, the distance between each PVIS, each PVRC, and each TC, and the size of each PVIS and the annual capacity of each PVRC and TC. First, in the cost charge aspect, the total transportation cost consists of the overhead charge, the mileage charge, and the weight charge. The overhead charge (\$/trip) is a fixed expense charged by each trip. The mileage charge is the product of the unit mileage charge $(\$ / \mathrm{km})$ and the mileage $(\mathrm{km})$ travelled. The weight charge is the product of the unit weight cost $(\$ / \mathrm{kg})$ and the weight of the material $(\mathrm{kg})$ transported. The total cost of each transportation service is the sum of the overhead charge, the mileage charge, and the weight charge. The second input is the distance matrices consisting of a set of distance combination among PVIS, TC, and PVRC. With the latitude and the longitude of each PVIS, TC, and PVRC, the distance combination between two of three locations can be calculated and expressed in matrix forms. The third input is the size of each PVIS, TC, and PVRC. The weight of material of each PVIS affects the weight cost. The annual capacity of each TC and PVRC as a physical constraint affects the distribution result.
Two types of optimization algorithms were used in the modeling framework: a static optimization algorithm, and a dynamic optimization algorithm.

\section{Static optimization algorithm}

The static optimization algorithm is a type of the GA. GA is an algorithm of finding the optimal solution by using a heuristic, which orders inputs by a seemingly logical way. In theory, as long as all inputs are ordered in the special way, the GA makes the same decisions that the optimal solution does. In practice, the optimal solution obtained by the GA is most likely a local optimum. However, the local optimum solution is sometimes very close to the global optimum with the reduction of significant amount of running time.

In the model, all PVIS can be sorted by their sizes from the largest to the smallest. The explanation is that the larger PVIS are the ones that would save the most money by choosing the cheapest transportation option. After sorting the size of PVIS, the largest PVIS has the first priority to be recycled by the first TC that offers the cheapest price. The less size PVIS has, the later that PVIS will be considered by the GA. As a result, for PVIS with larger sizes, the cost on transportation of those is minimal. Though the saving of each PVIS is small, the total saving can accumulate by considering some thousands of PVIS. The annual capacities of PVRC and TC were included in the model as constraints.

\section{Dynamic optimization algorithm}

The dynamic optimization algorithm solves the problem by using the MILP. Eq. (1) is the objective function to minimize the total transportation cost. Again, the total transportation cost is the sum of the overhead charge, the mileage charge, and the weight charge. Each type of the charge was described in the previous section. Eq. (2) is to ensure all installed PVIS are sent as a whole to one of the PVRC. The linear inequalities in Eq. (3) restrict that the total weight that each PVRC receives is less than its maximum capacity. The linear inequalities in Eq. (4) ensure that the total weight of each TC transports is less than its maximum transportation capacity. The model iterates until it identifies the minimum total transportation cost.

Minimize

$$
\begin{gathered}
\sum_{i \in I} \sum_{j \in J} \sum_{k \in K}\left\{\left[o_{k} \times n_{i}+m_{k} \times\left(D_{k i}+D_{i j}+D_{j k}\right)\right.\right. \\
\left.\left.\times n_{i}+w_{k} \times W_{i}\right] \times Y_{i j k}\right\}
\end{gathered}
$$




$$
\begin{aligned}
& \sum_{j \in J} \sum_{k \in K} Y_{i j k}=1 \\
& \sum_{i \in I} \sum_{k \in K} W_{i} Y_{i j k} \leq C_{j} \\
& \sum_{i \in I} \sum_{j \in J} W_{i} Y_{i j k} \leq C_{k}
\end{aligned}
$$

where, subscripts $i, j$, and $k$ represent PVIS, PVRC, and TC, respectively. $o_{k}, m_{k}$, and $w_{k}$ is the overhead charge, the unit mileage charge, and the unit weight charge that TC $k$ offeres, respectively. $n_{i}$ is the number of required trips to transport PVIS $i$. $D_{k i} D_{i j}$, and $D_{j k}$ are the transportation distances between corresponding locations. $W_{i}$ is the weight of material of PVIS $i . Y_{i j k}$ is a binary decision factor: if PVIS $i$ is transported to PVRC $j$ by TC $k$, then $Y_{i j k}=1$, otherwise, $Y_{i j k}=0 . C_{j}$ and $C_{k}$ is the maximum annual recycling capacity of PVRC $j$ and TC $k$, respectively.

\section{Experiment}

A case study in California is considered to show the efficacy of the proposed framework. The State of California was selected because of its relatively long history and the significant amount of PV installations compared to other states. In the case study, three recycling periods and several PVRC locations were assumed based on preliminary studies. In those studies, three recycling periods were recommended for recycling the EoL PV between the years 1984 and 2015 based on the amount of EoL PV as well as the increasing trend in each year. The location as well as the annual capacity of PVRC in each period was recommended as well: two-2.6 kt annual capacity PVRC in Period I, and addition 6-8 kt in Period II and Period III, respectively. The above assumptions were utilized for implementing the optimization model.

The annual PV installation of California is shown in Fig. 1. The figure shows the number of PVIS of each size range, as well as their size percentage among the total size of each year. Three recycling periods were divided. The first period has fewer PVIS, as well as a small amount of $\mathrm{kW}$. The second period is the next. And the third period has the most PVIS, as well as the most amount of kW. Another insight of Fig. 1 is the number of PVIS in each $\mathrm{kW}$ range is not proportional to its total $\mathrm{kW}$ percentage. For instance, the size range of $3-10 \mathrm{~kW}$ contains the most PVIS (>70\%) from the year 2009 to 2012. However, the total $\mathrm{kW}$ of that size range of PVIS is less than $30 \%$. In the opposite, the number of PVIS in size range of $100-1200 \mathrm{~kW}$ seems negligible. However, they account for about $50 \%$ of the total kW amount. In conclusion, the small size (3-10 $\mathrm{kW}$ ) PVIS are dominated in the quantity perspective, but in the overall amount of $\mathrm{kW}$ perspective, the proportion of larger size (100-2500 kW) PVIS should not be negligible.

Considering the gigantic amount of PVIS in California, the timing of collection should be considered while operating and managing the recycling process in reality. The framework assumed all EoL PVIS have been disassembled and are ready to load on the truck. In addition, the following assumptions are made: the maximum load of a truck is $7.5 \mathrm{t}$, and the approximate weight of material of every 1 MW capacity PV modules is equivalent to $75 \mathrm{t}$ [14]. With the above assumptions, each truck can take at most 100 kW PV modules. Considering the significant percentage (about 40\%, in Fig. 1) of PV modules that are over 100 $\mathrm{kW}$, the number of trips cannot be ignored.

Further reasonable assumptions are made to simplify the optimization process. Due to the gigantic number of PVIS (up to 160,000 PVIS per year) together with the combinational delivering options between PVIS, PVRC, and TC, reasonable simplifications need to make to satisfy the computational requirement on both software and hardware. Thus, another two reasonable assumptions are made. The first is the unit cost charge similarity assumption. In reality, the cost charge by each TC tends to be similar due to the competitive market. Therefore, the unit cost charge of each $\mathrm{TC}$, such as $\$ /$ trip, $\$ / \mathrm{km}$, and $\$ / \mathrm{kg}$, are assumed to be the same. The second is the dead mileage charge exemption assumption. In fact, TC usually do not charge on
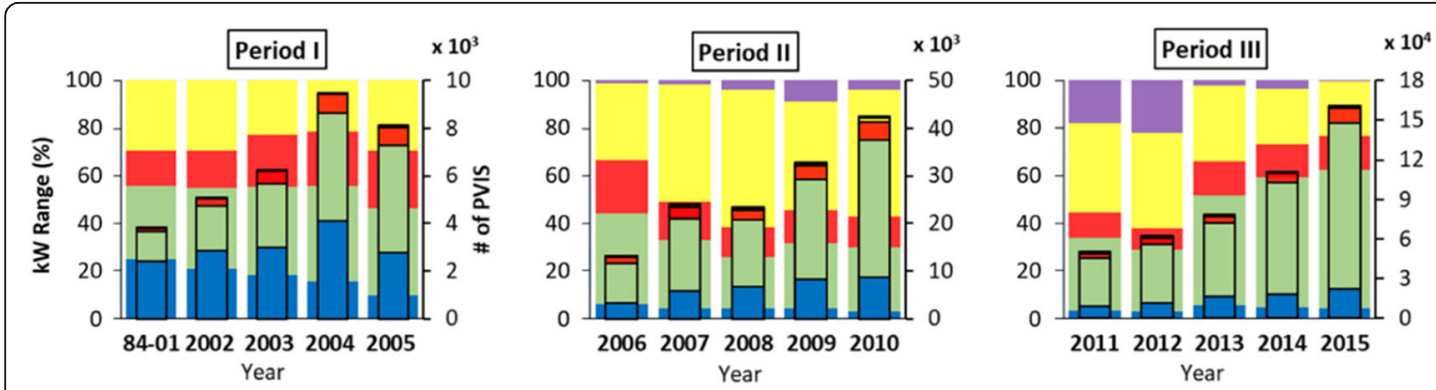

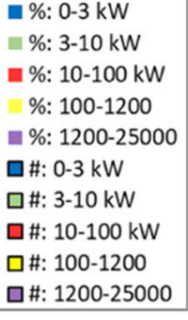

Fig. 1 Annual PV installations in California (1984-2015) 
dead mileage. The dead mileage is the mileage generation when vehicles are driven on the way to or back from the worksite without taking the real workload. In the case study, the dead mileage is the mileage when trucks driving from TC to PVIS, and the mileage when trucks driving from PVRC to TC. Those dead mileage charges are reasonable to be exempted in the case study by considering the reality. With the cost charge similarity assumption and the dead mileage charge exemption assumption, TC is no longer a variable in the optimization process. Subsequently, the transportation cost function can be simplified from Eq. (1) to Eq. (5). The simplified transportation cost function consists of the overhead charge, $o$, the unit mileage charge $m$, the unit weight charge, $w$, the travel distance between the PVIS and the PVRC, $D$, the weight of material of the PVIS, $W$, and the number of trips, $n$. By using the simplified cost function, variables in optimization processes can be reduced by at least $50 \%$.

Transportation cost $=o \times n+m \times D \times n+w \times W$

With the simplification, the dynamic optimization algorithm is simplified below.

Minimize

$$
\sum_{i \in I} \sum_{j \in J}\left\{\left[o \times n_{i}+m \times D_{i j} \times n_{i}+w \times W_{i}\right] \times Y_{i j}\right\}
$$

Subject to

$$
\begin{aligned}
& \sum_{j \in J} Y_{i j}=1 \\
& \sum_{i \in I} W_{i} Y_{i j} \leq C_{j}
\end{aligned}
$$

The simplified form can improve the feasibility for handling large numbers of PVIS and fasten the computational speed of MILP. In addition, rather than MILP, Linear Program (LP) is recommended and utilized for solving the particular California case. It is concluded that LP is able to handle more variables, compute and converge much faster, and conduct the total transportation cost with the error of less than $1 \%$ of the total transportation cost conducted by the MILP.

While performing the optimization, reasonable unit charges were assumed. The real market price of unit charges was not used as a main focus in the paper because those prices vary from time by time. All the assumed prices were for initiating the process and showing the efficacy of the PV recycling framework. Thus, the following unit costs were utilized: the overhead cost is $\$ 10 /$ trip, the unit mileage cost is $\$ 0.06 / \mathrm{km}$, and the unit weight cost is $\$ 0.008 / \mathrm{kg}$. The framework can be employed in any multi-facility recycling problem anywhere as long as the unit cost rates are available.

\section{Results and discussion}

Matlab was utilized as the tool to perform the optimization for both algorithms. The baseline total transportation cost, and the optimal total transportation cost conducted by the GA and the LP are shown in Fig. 2. The baseline cost indicates the total transportation cost of recycling PVIS by their installed date without doing any optimization. In that way, each PVIS is going to the closest PVRC whose maximum annual capacity has not been reached. The transportation cost conducted by GA and LP is the optimal cost as they are generated by optimization processes. The mechanism of the GA is recycling PVIS from the largest size to the smallest, while LP processes the optimization with the objective of minimizing the overall transportation cost.

Ideally, LP provides the lowest optimal cost, but also with the most computational expense. The optimal cost that GA provided is higher than LP, but lower than the baseline cost. However, in Fig. 2, GA even costs more than baseline. The discrepancy is due to the specialty of the size distribution of CA PVIS as well as the effect of the hypothesis cost structure. Table 1 shows the combination of transporting the total 100, 300, and $500 \mathrm{~kW}$ PVIS for $100 \mathrm{~km}$. In that, 100 of $1 \mathrm{~kW}, 10$ of $10 \mathrm{~kW}$, and 1 of $100 \mathrm{~kW}$ of PVIS were used for the total $100 \mathrm{~kW}$ and the same logic for the total 300 and $500 \mathrm{~kW}$ of PVIS. The cost multiplier in the last column of Table 1 indicates the higher number of cost associated with the option of a single PVIS unit. Table 1 shows that due to the large percentage of the overhead cost among the grand total, the cost of transporting 100 of $1 \mathrm{~kW}$ is 22 times more expensive than transporting 1 of $100 \mathrm{~kW}$, and 8 and 5 times more expensive for the 300 and $500 \mathrm{~kW}$ case, respectively. Thus, small size PVIS should be considered first for saving the most money. In addition, from Fig. 1, the number of PVIS with the size in between 0 and $10 \mathrm{~kW}$ accounts for about $90 \%$ of the total. In conclusion, for recycling PVIS in CA with the current hypothesis cost structure, having small PVIS recycled in the cheapest way will maximize the total transportation cost saving. Therefore, GA should be modified as sorting the size of PVIS from the smallest to the largest and assign the cheapest transport option to smaller PVIS first and is denoted as GA'.

Table 2 shows the transportation cost savings by the modified GA (GA') and LP of each year. There are three 


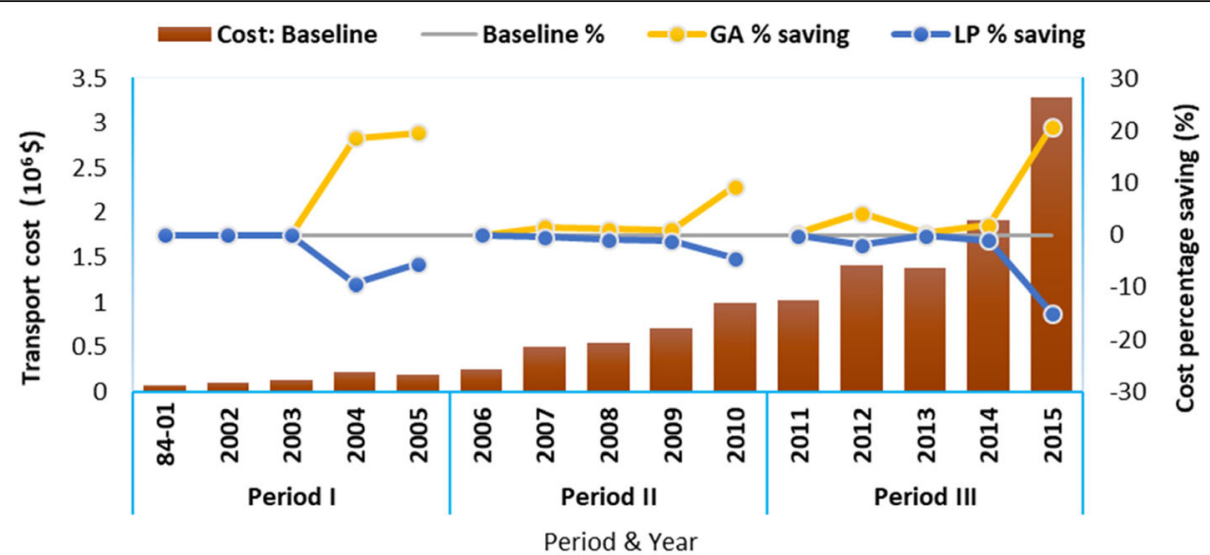

Fig. 2 Transportation cost and cost percentage saving by each method and year

conclusions. First, saving only appears in those years when the annual capacity of PVRC is relatively tight compared with the amount of PV that needs to be recycled (amount of installed PV). The tighter annual capacity of PVRC, the more saving is expected by doing optimization. No savings when the annual capacity of PVRC is about two or more times than the amount of PV that needs to be recycled. Second, the saving differences between GA' and LP in each scenario are trivial. However, the computational of LP could be much longer than GA', especially when the number of both PVIS and PVRC increases significantly. The computation time of LP for the year 2015 is as high as $3.5 \mathrm{~h}$. Third, the number of PVIS is another factor that affects the saving. The amount of installed PV in the year of 2004 is less than that in 2005. However, the saving of the year 2004 is twice of the that in 2005. This difference is because the number of PVIS in the year of 2004 is about one third more than that in 2005 as shown in Fig. 1. Based on the above conclusion, in the CA case, it is reasonable to rely on the solution generated by $\mathrm{GA}^{\prime}$ with a relative accurate solution, a short running time than LP, without purchasing an optimization solver.

From Table 2, though the saving is as high as $\$ 491,000$ in the year 2015 , the percentage saving is only $15 \%$, which is not significant. The reason of the insignificance results from the category of the charges in the current cost function. The current cost function is shown in Eq. (5), and is denoted as Cost Structure 1. Cost Structure 1 is composed of the overhead charge, the weight cost, and the mileage charge. In Cost Structure 1, the overhead charge (\$/trip) and the weight cost are not optimized and remain the same based on the unit cost charge similarity assumption. The weight cost is identical because the unit weight cost $(\$ / \mathrm{kg})$ is identical from the unit cost charge similarity assumption. Therefore, the mileage charge is the only parameter that determines the total cost saving. The next step is creating another cost structure, Cost Structure 2, which is only in terms of the mileage charge as shown in Eq. (9). The transportation cost in Cost Structure 2 only consists of the unit mileage cost, $m$, the travel distance,

Table 1 Total cost and multiple of cost of PVIS size and number of PVIS combination

\begin{tabular}{|c|c|c|c|c|c|c|c|c|}
\hline $\begin{array}{l}\text { PVIS size } \\
(\mathrm{kW})\end{array}$ & $\begin{array}{l}\text { Number of } \\
\text { trips }\end{array}$ & $\begin{array}{l}\text { Overhead cost } \\
\text { (\$) }\end{array}$ & $\begin{array}{l}\text { Mileage cost } \\
(\$)\end{array}$ & $\begin{array}{l}\text { Weight cost } \\
\text { (\$) }\end{array}$ & $\begin{array}{l}\text { Grand total } \\
\text { (\$) }\end{array}$ & $\begin{array}{l}\text { Number of } \\
\text { PVIS }\end{array}$ & $\begin{array}{l}\text { Total cost } \\
(\$)\end{array}$ & $\begin{array}{l}\text { Cost } \\
\text { multiplier }\end{array}$ \\
\hline 1 & 1 & 10 & 6 & 1 & 17 & 100 & 1660 & $22 x$ \\
\hline 10 & 1 & 10 & 6 & 6 & 22 & 10 & 220 & $3 x$ \\
\hline 100 & 1 & 10 & 6 & 60 & 76 & 1 & 76 & 1 \\
\hline 3 & 1 & 10 & 6 & 2 & 18 & 100 & 1780 & $8 x$ \\
\hline 30 & 1 & 10 & 6 & 18 & 34 & 10 & 340 & $1 x$ \\
\hline 300 & 3 & 30 & 18 & 180 & 228 & 1 & 228 & 1 \\
\hline 5 & 1 & 10 & 6 & 3 & 19 & 100 & 1900 & $5 x$ \\
\hline 50 & 1 & 10 & 6 & 30 & 46 & 10 & 460 & $1 x$ \\
\hline 500 & 5 & 50 & 30 & 300 & 380 & 1 & 380 & 1 \\
\hline
\end{tabular}


Table 2 Saving of $G A^{\prime}$ and LP of each year

\begin{tabular}{|c|c|c|c|c|c|c|}
\hline Period & $\begin{array}{l}\text { PVRC recycling capacity } \\
\text { (MW/yr) }\end{array}$ & $\begin{array}{l}\text { Installed } \\
\text { year }\end{array}$ & $\begin{array}{l}\text { Amount of installed } \\
\text { PV (MW) }\end{array}$ & $\begin{array}{l}\text { GA' saving } \\
\left(10^{3} \$\right)\end{array}$ & $\begin{array}{l}\text { LP saving } \\
\left(10^{3} \$\right)\end{array}$ & $\begin{array}{l}\text { Computing time ratio } \\
\left(\mathrm{LP} / \mathrm{GA}^{\prime}\right)\end{array}$ \\
\hline \multirow[t]{5}{*}{ I } & 70 & 84-01 & 19 & 0 & 0 & 11 \\
\hline & & 2002 & 29 & 0 & 0 & 15 \\
\hline & & 2003 & 40 & 0 & 0 & 14 \\
\hline & & 2004 & 63 & 20 & 20 & 15 \\
\hline & & 2005 & 65 & 10 & 10 & 15 \\
\hline \multirow[t]{5}{*}{$\|$} & 700 & 2006 & 113 & 0 & 0 & 70 \\
\hline & & 2007 & 287 & 2 & 2 & 152 \\
\hline & & 2008 & 346 & 5 & 5 & 148 \\
\hline & & 2009 & 418 & 8 & 9 & 235 \\
\hline & & 2010 & 603 & 44 & 45 & 311 \\
\hline \multirow[t]{5}{*}{ III } & 1300 & 2011 & 643 & 2 & 2 & 869 \\
\hline & & 2012 & 953 & 26 & 27 & 1187 \\
\hline & & 2013 & 671 & 3 & 3 & 1506 \\
\hline & & 2014 & 866 & 20 & 22 & 2544 \\
\hline & & 2015 & 1216 & 473 & 491 & 3731 \\
\hline
\end{tabular}

$D$, and the number of trips, $n$. Assume the unit mileage cost is $\$ 0.2 / \mathrm{km}$.

$$
\text { Transportation cost }=m \times D \times n
$$

Table 3 shows the saving comparison that generated by different cost structure of each year. From the table,

Table 3 Percentage saving of in each cost structure of each year. (Unit: \%)

\begin{tabular}{|c|c|c|c|c|c|}
\hline \multirow[t]{2}{*}{ Period } & \multirow{2}{*}{$\begin{array}{l}\text { Installed } \\
\text { year }\end{array}$} & \multicolumn{2}{|c|}{ Cost structure 1} & \multicolumn{2}{|c|}{ Cost structure 2} \\
\hline & & GA' saving & LP saving & GA' saving & LP saving \\
\hline \multirow[t]{5}{*}{ I } & $84-01$ & 0 & 0 & 0 & 0 \\
\hline & 2002 & 0 & 0 & 0 & 0 \\
\hline & 2003 & 0 & 0 & 0 & 0 \\
\hline & 2004 & 9 & 9 & 24 & 25 \\
\hline & 2005 & 5 & 6 & 16 & 16 \\
\hline \multirow[t]{5}{*}{$\|$} & 2006 & 0 & 0 & 0 & 0 \\
\hline & 2007 & 0 & 0 & 2 & 3 \\
\hline & 2008 & 1 & 1 & 6 & 6 \\
\hline & 2009 & 1 & 1 & 7 & 8 \\
\hline & 2010 & 4 & 5 & 25 & 26 \\
\hline \multirow[t]{5}{*}{ III } & 2011 & 0 & 0 & 1 & 1 \\
\hline & 2012 & 2 & 2 & 15 & 16 \\
\hline & 2013 & 0 & 0 & 2 & 2 \\
\hline & 2014 & 1 & 1 & 8 & 8 \\
\hline & 2015 & 14 & 15 & 51 & 53 \\
\hline
\end{tabular}

savings turns to be significant, at most $50 \%$, especially when the annual capacity of PVRC is tight. Therefore, the transportation optimization is beneficial.

The color-coded distribution decision by GA' is displayed on maps by GIS as shown in Fig. 3. The installed years 2003, 2008, and 2013 were selected to represent the installed period I, II, and III, respectively. PVIS were assigned to the closest, non-full PVRC, represented as each identical color of PVIS and PVRC.

\section{Conclusions}

A generic mathematical framework was constructed to generate the optimal distribution scheme for transporting EoL PVIS. A static algorithm (GA) and a dynamic optimization algorithm (LP) were employed in the framework. The following conclusions were made from the simulation result based on the PV installed case in CA. First, based on the size distribution of PV installed in CA, the focus of the optimization should be on small PVIS rather than large ones. The most saving on transportation cost is obtained by optimizing transport distribution on small PVIS. Second, GA is a reliable algorithm to perform the optimization on transportation cost with decent accuracy, low computational cost, and no optimization solver required for the CA case. Third, the transportation cost saving increases with the annual capacity of PVRC getting tighter. With the appropriate optimization process and the specific cost structure applied, the transportation cost savings can be expected at 53\% compared with 

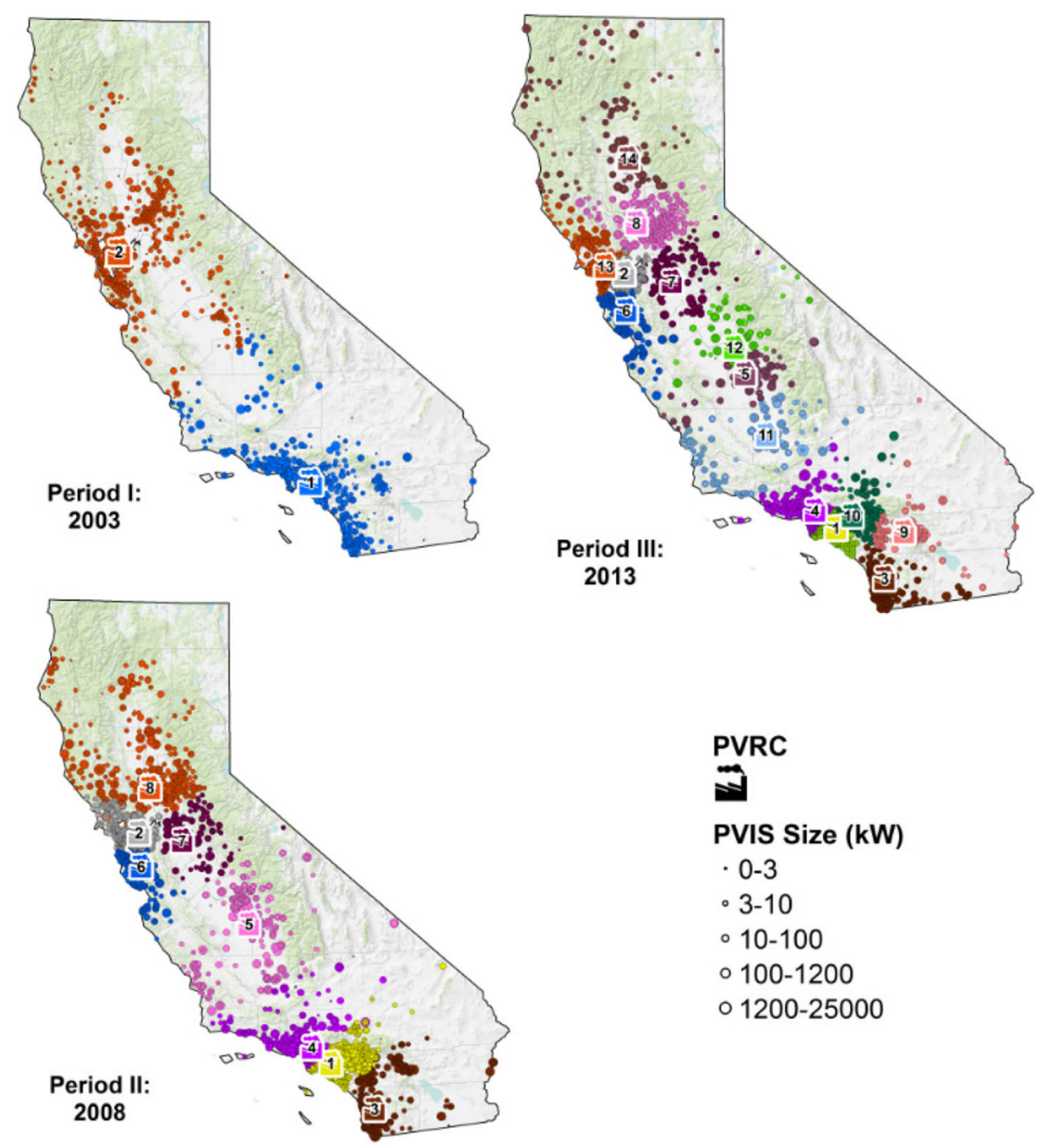

Fig. 3 Distribution decision of year 2003, 2008, and 2013

not doing optimization. When the annual recycling capacity of PVRC is twice or larger than the amount of the recycled PV demand, there will not be transportation savings, and thus no optimization is needed. The developed framework has a wide applicability and can be applied on any PV recycling case for reducing the transportation cost.

Authors' contributions

Both authors read and approved the final manuscript.

\section{Competing interests}

The authors declare that they have no competing interests.

\section{Publisher's Note}

Springer Nature remains neutral with regard to jurisdictional claims in published maps and institutional affiliations.

\section{Author details}

'Department of Engineering Technologies, Bowling Green State University, Bowling Green, Ohio 43402, USA. ${ }^{2}$ Guizhou Kaiyang Chemical Industry Co., Ltd, Yongwen 550306, China.

Received: 8 August 2018 Accepted: 26 November 2018 Published online: 19 March 2019

\section{References}

1. SPE. Global Market Outlook for Solar Power 2016-2020. Brussels: SolarPower Europe; 2016.

2. Perea A, Honeyman C, Kann S, Mond A, Shiao MJ, Jones J, et al. U.S. Solar Market Insight: 2016 Year in Review. Edinburgh and Washington, DC: Wood Mackenzie, Limited and Solar Energy Industries Association; 2017.

3. Choi JK, Fthenakis V. Design and optimization of photovoltaics recycling infrastructure. Environ Sci Technol. 2010:44:8678-83.

4. Hosenuzzaman M, Rahim NA, Selvaraj J, Hasanuzzaman M, Malek ABMA, Nahar A. Global prospects, progress, policies, and environmental impact of solar photovoltaic power generation. Renew Sust Energ Rev. 2015;41:284-97.

5. Choi JK, Fthenakis V. Crystalline silicon photovoltaic recycling planning: macro and micro perspectives. J Clean Prod. 2014;66:443-9. 
6. Goe M, Gaustad G, Tomaszewski B. System tradeoffs in siting a solar photovoltaic material recovery infrastructure. J Environ Manag. 2015;160: 154-66.

7. Cucchiella F, D'Adamo I, Rosa P. End-of-life of used photovoltaic modules: a financial analysis. Renew Sust Energ Rev. 2015;47:552-61.

8. Göllei A, Görbe P, Magyar A. Modeling and optimization of electrical vehicle batteries in complex clean energy systems. J Clean Prod. 2012;34:138-45.

9. Daskin MS, Snyder LV, Berger RT. Facility location in supply chain design. In: Langevin A, Riopel D, editors. Logistics systems: design and optimization. Boston: Springer; 2005. p. 39-65.

10. Akhtar M, Hannan MA, Begum RA, Basri H, Scavino E. Backtracking search algorithm in CVRP models for efficient solid waste collection and route optimization. Waste Manag. 2017;61:117-28.

11. Kallel A, Serbaji MM, Zairi M. Using GIS-based tools for the optimization of solid waste collection and transport: case study of Sfax City, Tunisia. J Eng. 2016;2016:1-7.

12. Demirel $\mathrm{E}$, Demirel $\mathrm{N}$, Gökçen $\mathrm{H}$. A mixed integer linear programming model to optimize reverse logistics activities of end-of-life vehicles in Turkey. J Clean Prod. 2016;112:2101-13.

13. Qin JJ, Chow YL, Yang JY, Rajagopal R. Distributed online modified greedy algorithm for networked storage operation under uncertainty. IEEE T Smart Grid. 2016;7:1106-18.

14. Choi JK, Fthenakis V. Economic feasibility of recycling photovoltaic modules. J Ind Ecol. 2010;14:947-64.

Ready to submit your research? Choose BMC and benefit from:

- fast, convenient online submission

- thorough peer review by experienced researchers in your field

- rapid publication on acceptance

- support for research data, including large and complex data types

- gold Open Access which fosters wider collaboration and increased citations

- maximum visibility for your research: over $100 \mathrm{M}$ website views per year

At $\mathrm{BMC}$, research is always in progress.

Learn more biomedcentral.com/submissions 